Supporting Information for

\title{
In-Situ Nanotribological Properties of Ultrananocrystalline Diamond Films Investigated with Ambient Pressure Atomic Force Microscopy
}

Jae-Eun Kim, ${ }^{\dagger,}$ Joong Il Jake Choi,${ }^{\dagger}$ Jeongjin Kim,${ }^{\dagger}$ Bongjin Simon Mun, ${ }^{\ddagger}$ Ki-Jeong Kim, ${ }^{\| ~ \& ~}$ Jeong Young Park ${ }^{\dagger},, *$

${ }^{\dagger}$ Center for Nanomaterials and Chemical Reactions, Institute for Basic Science (IBS), Daejeon 34141, Republic of Korea

$\S$ Department of Chemistry, Korea Advanced Institute of Science and Technology (KAIST), Daejeon 34141, Republic of Korea

* Department of Physics and Photon Science, School of Physics and Chemistry, Gwangju Institute of Science and Technology (GIST), Gwangju 61005, Republic of Korea

" Beamline Research Division, Pohang Accelerator Laboratory (PAL), POSTECH, Pohang 37673, Republic of Korea

\section{KEYWORDS}

tribology, friction, ultrananocrystalline diamond, ambient pressure $x$-ray photoelectron spectroscopy, surface passivation 


\section{Explanation of Load-Dependent Friction Behavior with JKR and DMT Models}

In Equation (1), $K$ is the reduced Young's modulus, in which $v_{\mathrm{t}}$ and $v_{\mathrm{s}}$ are the Poisson ratio, and $E_{t}$ and $E_{s}$ are Young's moduli of the tip and sample, respectively. Equation (2) describes the relationship between the contact area $(A)$ and applied load $(L)$ of compliant materials, and the model is called JKR (Johnson-Kendall-Roberts) model. ${ }^{1}$ Also, in this model, the adhesion force $\left(L_{\mathrm{ad}}\right)$ is relevant to the work of adhesion $(\gamma)$, by the equation (3). In DMT (Derjaguin-MullerToporov) model, ${ }^{2}$ for the hard and less adhesive materials, the relationship between the $A$ and $L$, and the relationship between $L_{\mathrm{ad}}$ and $\gamma$ follow Equation (4) and (5), respectively.

$$
\begin{aligned}
& \frac{1}{K}=\frac{3}{4}\left(\frac{1-v_{s}^{2}}{E_{s}}+\frac{1-v_{t}^{2}}{E_{t}}\right) \\
& A=\pi\left\{\frac{R}{K}\left[L+3 \pi R \gamma+\left(6 \pi R \gamma L+(3 \pi R \gamma)^{2}\right)^{1 / 2}\right\}^{2 / 3}\right. \\
& L_{\text {ad }}=\frac{3}{2} \pi R \gamma \\
& A=\pi\left\{\frac{R}{K}(L+2 \pi R \gamma)\right\}^{2 / 3} \\
& L_{\text {ad }}=2 \pi R \gamma
\end{aligned}
$$



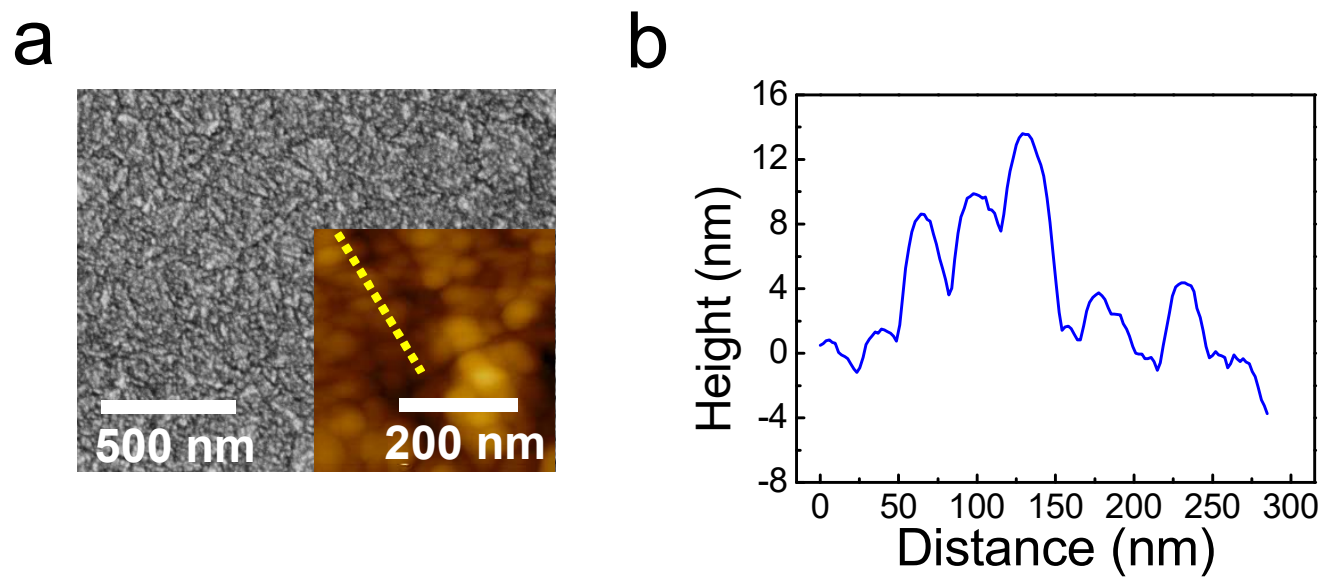

Figure S1. (a) Morphological images of UNCD by FE-SEM and NC-AFM (inset). (b) Line profile of yellow dot line of the inset of (a). The root-mean-square roughness of UNCD is $\sim 8 \mathrm{~nm}$, and the grain size is less than $30 \mathrm{~nm}$, as can be seen in the profile. 


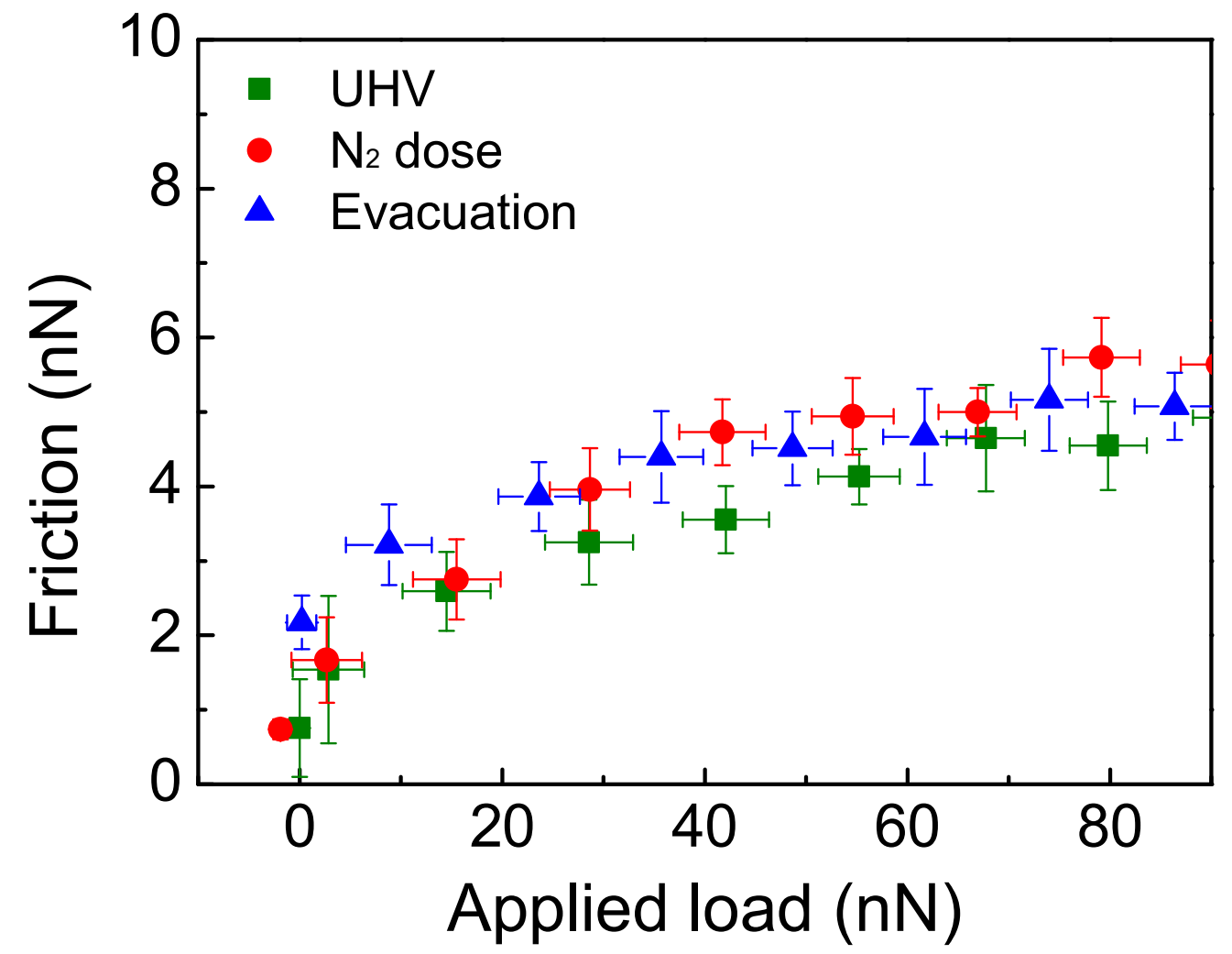

Figure S2. Load dependence of frictional behavior of UNCD measured by FFM at UHV (2.0x1010 mbar), $\mathrm{N}_{2}$ pressure of 154 mbar and after evacuation $\left(2.8 \times 10^{-8}\right.$ mbar). $\mathrm{N}_{2}$ did not affect the friction of UNCD due to its inertness. 


\section{REFERENCES}

1. Johnson, K. L.; Kendall, K.; Roberts, A. D., Surface Energy and the Contact of Elastic Solids. Proceedings of the Royal Society A 1971, 324, 301.

2. Derjaguin, B. V.; Muller, V. M.; Toporov, Y. P., Effect of Contact Deformation on the Adhesion of Particles. Journal of Colloid and Interface Science 1975, 53, 314. 